\title{
Performance of Movable Head Disk Storage Devices with Various Disk Scheduling Algorithms
}

\section{A Gomathy}

\begin{abstract}
Hard drives are the one which needs to be accessed in an efficient manner so that it is feasible to get better recital of the central processing unit. Now a day's magnetic disks are capable of providing more input output bandwidth yet a huge amount of this bandwidth is lost due to the access time of the hard disk. This paper discusses an analysis of performance of various disk scheduling algorithms with their merits and demerits

Index Terms: Fcfcs, Sstfs, Scans, C-Scans, F-Scans, Looks, C-Looks, S-Looks
\end{abstract}

\section{INTRODUCTION}

Controlling the disk performance is an imperative role of an operating system. Now a day the velocity of the processor and the main memory has been improved quite a lot of times than the speed of the secondary memory especially hard disk. This divergence of the speed of the processor and the hard disk leads to the input/output performance of the disk to the question. In any disk structure with a moving read and write head, the seeking time to reach the desired cylinders and sectors takes a considerable quantity of time. This seeking time should be minimized to get enhanced access time. Using the computer hardware in an efficient manner must be the major responsibility of an operating system

\section{A. Importance of Disk Scheduling}

- Hard drives require to be accessed in an efficient manner since it is one of the slowest parts of the CPU.

- Multiple input and output requests possibly will raise by various processes and no more than one input and output request can be served at a time by means of the disk controller. As a result the further input and output requests must be in the waiting queue and these requests have to be scheduled.

- Two or more request possibly will be so far from each other so it may perhaps result in higher disk arm movement.

\section{B. Important Parameters in Disk Scheduling}

1. Look for Time: Time spent to locate the diskette armrest to a precise track where the data is to be read or write is located in fact is known as look for time.

2. Rotational Latency: Time taken by chosen sector to swivel into position so that it can be accessed by the read or write head is known as rational latency.

3. Transfer Time: Time taken to transfer the data is known as transfer time. On the whole it varies depends on the speed of the hard disk and overall amount of bytes to be transfer

Revised Manuscript Received on December 15, 2019.

Gomathy A, Department of Computer Science and Engineering, Hindusthan College of Engg and Tech., Coimbatore, India.

Email:gomathy.aucbe@gmail.com

It can be defined as

$T T=B / S N$

Where $T T=$ Data Transfer Time

$B=$ Amount of data to be transferred (in bytes)

$N=$ Overall amount of data on a track (in

bytes)

$\mathrm{S}=$ Speed (in RPS-Rotation per Second)

4. Response Time: The standard time worn-out before a request is serviced.

5. Diskette Bandwidth: The disk bandwidth is the amount of data(in bytes) to be transferred divided by the overall time between the initial request for service and the completion of the final transfer.

6. Throughput : It can be defined as the number of requests serviced for each unit of time.

7. Diskette Access Time: It can be defined as the sum of seek time, rotational latency and transfer time.

8. Diskette reaction time: This can be defined as the average time exhausted by a process to perform its operation.

\section{Looking for optimal Algorithm}

The optimization factor we selected for this study to select the optimum algorithm is the look for time. It is defined the same as the time taken to locate the disk arm to a precise track where the read or write operation needs to be performed.

A scheduling algorithm should maximize the throughput and minimize the mean response time. We desire a scheduling principle that minimizes service time. Disk scheduling algorithms are used to prefer any one of the disk requests for read or write operation on hand to execute.

\section{DISK SCHEDULING PRINCIPLES}

We consider a disk with 200 tracks numbered as 0 through 199 and the number of processes raising requests in unsystematic manner. The following queue shows the order of read or write request to the corresponding cylinder numbers.

I/O Request queue: $23,89,132,42,187$

We consider that the present position of the disk head is at 100 and the disk head going inward that is towards 0 . With the seeking rate of $3 \mathrm{~ms}$, number of head movements and the overall seek time required to serve all the requests have to be calculated and compared for diverse scheduling principles.

\section{A. Fst.Come Fst. Serve (F-C-F-S) Scheduling} Principle:

FCFSS is the straightforward type of disk scheduling principle. 
This principle uses a "first in first serve" queue as a result the requests are serviced in the identical order in which they arrived. In this principle all the input and output requests are picked from the queue in a linear or sequential order. Still this principle is commonly does not provide the greater response time.

\section{Solution:}

As per the principle of "first come first serve" scheduling the requests will be serviced in the same order in which they arrived to the request queue. With respect to the taken example it will examine the request for cylinder number 23 then cylinder number 89 and so on. Finally the request for cylinder number 187 will be serviced.

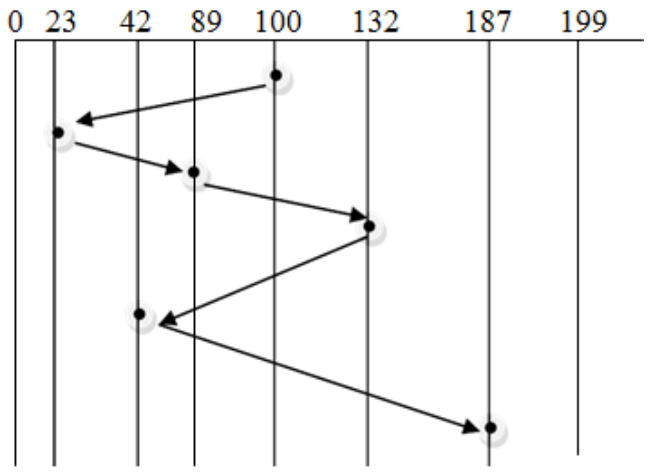

Figure.1.F-C-F-S Scheduling Principle

Overall head movements $=(100-23)+(89-23)+(132-$ $89)+(132-42)+(42-187)$ $=421$ cylinders

Overall seek time $=421 \times 3=1263 \mathrm{~ms}$

\section{B. Shortest .Seek Time. First (F-C-F-S) Scheduling Principle:}

S-S-T-F-S is a direct enhancement upon a "first come first serve" scheduling principle. The drive will maintain a queue of incoming requests along with their cylinder number. Minimum cylinder number indicates that the cylinder is nearer to the cylinder head while maximum cylinder number indicates the cylinder is farther than away.

The S-S-T-F scheduling principle determines which demand is nearby to the current position of the cylinder head, and makes the service of that one and then move on to the next. The "shortest seek time first" principle has the improvement compared to F-C-F-S-S principle. Taken as a whole the number of arm movement is reduced in S-S-T-F-S resulting in lesser response time. However, if the new request arises which is far from the current location leads to the starvation in servicing the new request.

\section{Solution:}

With respect to the "shortest seek time first" scheduling principle the request which is closer to the disk head will serviced first. As per the taken example the request for the cylinder number 89 is nearer to the disk head's present position that is 100 . And it will service to the next closest request and so on.

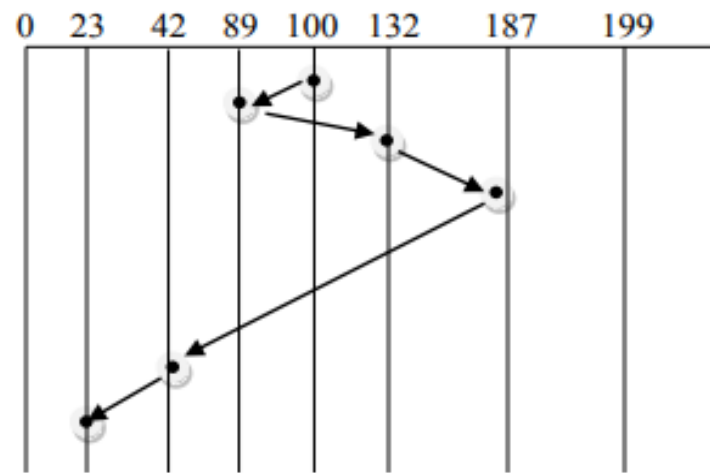

Figure.2. S-S-T-F Scheduling Principle

Overall head movements $=(100-89)+(132-89)+(187-$

$132)+(187-42)+(42-23)$

Overall seek time $=273 \times 3=819 \mathrm{~ms}$

$$
=273 \text { cylinders }
$$

\section{SCANS Scheduling Principle:}

According to SCANS scheduling principle, if a fresh request arrive when the hard drive is at leisure, the preliminary disk arm movement will be in the direction of the cylinder where the data is stored for the present request. This movement may be of either inward direction or outward direction. At the same time as further requests arrive, these new requests are serviced right away if those cylinders are in the identical direction of the current movement of the disk arm until the arm reaches the final cylinder of the disk. When the arm reaches the boundary of the disk, without delay the arm swings in the reverse direction and move towards the other end of the disk as well as it also serves every requests along the way. And it will continue the same until all the requests have been served.

\section{Solution:}

In "SCANS" scheduling principle all the requests in one direction will be served first and followed by it will be move on to the a further direction. Here in this example we considered as the disk head moves inward direction, initially it will move from 100 through 0 and it will serve all the requests along the way. Once it reaches cylinder number 0 the disk arm will adjust its direction to the reverse and it will serve the remaining requests.

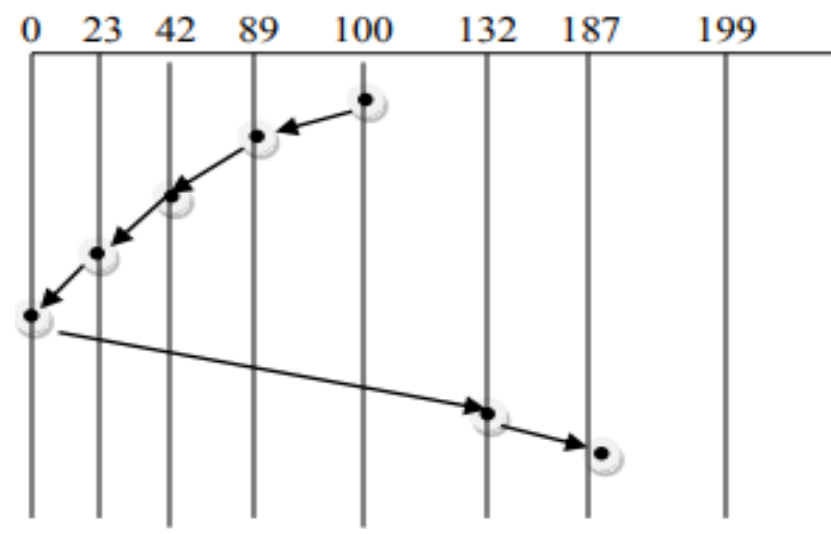

Figure.3.SCANS Scheduling Principle 
Overall head movements $=(100-89)+(89-42)+(42-23)$

$$
+(23-0)+(23-132)+(132-187)
$$

$$
=287 \text { cylinders }
$$

Overall seek time $=287 \times 3=861 \mathrm{~ms}$

Variants of SCANS Scheduling:

\section{B.1 Circular - SCAN Scheduling Principle:}

"Circular-SCAN" scheduling principle is an improved version of the "SCANS" scheduling principle. In "CSCAN" scheduling principle the disk head moves from the current position to the one end of the disk, it may possibly be of either inward direction or outward direction. During this journey it will serve all the requests along the way. When the head reaches one end of the disk straight away it will revert to the other end without servicing any requests being on the way of the return.

As per "CSCANS" principle the head moves from 100 through 0 and without delay it rolls back to 199 without servicing the request along the way.

\section{Solution:}

As per "CSCANS" scheduling principle the read or write head will move from 100 in the direction of the inward up to 0 and it will serve the entire requests on the way. Once it reaches cylinder number 0 it will swing to the other end of the disk i.e. 199 without servicing any request on the way as well as again it starts moving inward to reach 0 .

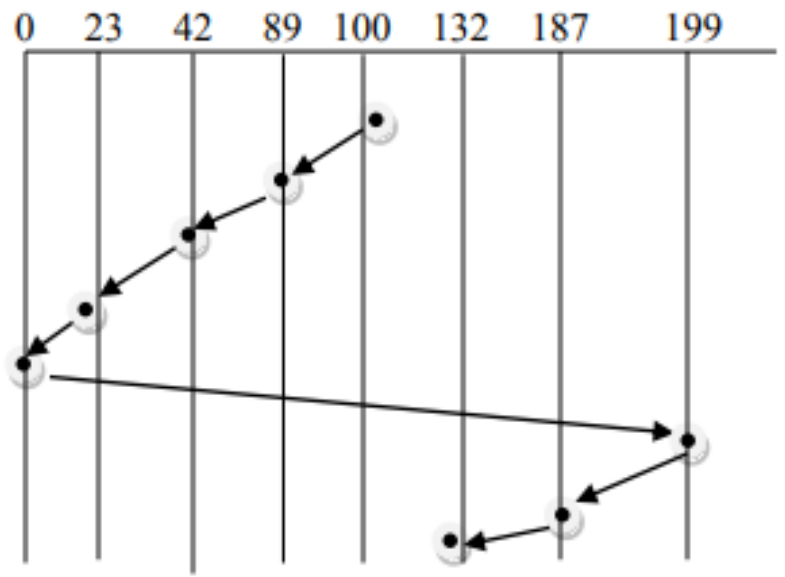

Figure.4. C-SCANS Scheduling Principle

Overall head movements $=(100-89)+(89-42)+(42-23)+$ $(23-0)+(199-0)+(199-187)+(187-132)$

$=366$ cylinders

Overall seek time $=366 \times 3=1098 \mathrm{~ms}$

\section{F-SCANS Scheduling Principle:}

F-SCANS scheduling principle determines the direction of the disk arm and the disk head movement in order to serve all the read and write requests. It uses two sub-queues. During the scan, all of the requests will be in the first queue and all new requests will be put into the second queue. Consequently, service of new request is overdue until all of the old requests have been processed. Once all the requests in the primary queue have been serviced, the arm will be taken to the secondary queue entries and all those will be serviced.

\section{E. LOOKS Scheduling Principle:}

The "LOOKS" scheduling principle is analogous to the "SCANS" scheduling principle in which it is also service the requests equally inward and outward swing of the disk head. Even so, this principle glance in advance to observe is there any request waiting in the direction of the head movement. In the direction of head movement if there is no requests are waiting, then the disk head traversal will be upturned to the contradictory direction and requests on the other direction can be served. The main divergence is that in "LOOKS" scheduling principle, the arm goes simply as far as the ultimate requested cylinder number in each direction and then changes the direction to the reverse direction exclusive of going to the end of the disk.

"LOOKS" scheduling principle behaves nearly identically to "shortest seeks time first" scheduling principle but avoids the starvation problem of S-S-T-F scheduling principle.

\section{Solution:}

As per "LOOKS" scheduling principle the read write head starts from cylinder number 100 and move towards the inward direction when it reaches the lowest cylinder number it starts moving to the other side of the disk up to the request for the highest cylinder. Corresponding to the example we considered it will move up to 187 moreover it will service the entire request along the way.

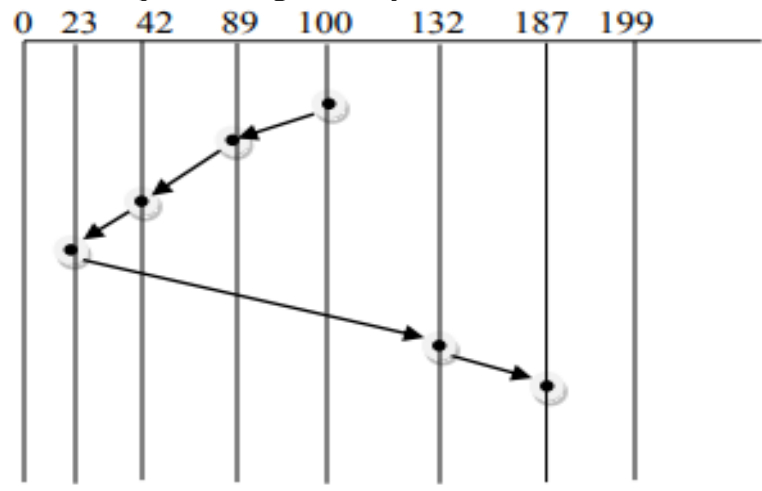

Figure.5. LOOKS Scheduling Principle

Overall head movements $=(100-89)+(89-42)+(42-23)$ $+(23-132)+(132-187)=241$ cylinders

Overall seek time $=241 \times 3=723 \mathrm{~ms}$

Variants of LOOKS Scheduling:

\section{E.1 Circular- LOOKS Scheduling Principle:}

One modification of "LOOKS" scheduling is circular LOOKS. It is an endeavor to eradicate the preconceived notion in LOOKS scheduling principle. Circular LOOKS on the whole scans in only one direction. Either sweep from inside to the out, or from the outside to the in. When the disk head reaches the end, it will dangle reverse to the beginning. This will be a merit in the situation that when many drives move the read/write head at high speeds across a large number of tracks. 


\section{Solution:}

As per Circular LOOKS scheduling principle when the disk head reaches cylinder number 23 from cylinder number 100 it will revert to the requested cylinder number which is located at the other end of the disk and not to the final cylinder of the disk.

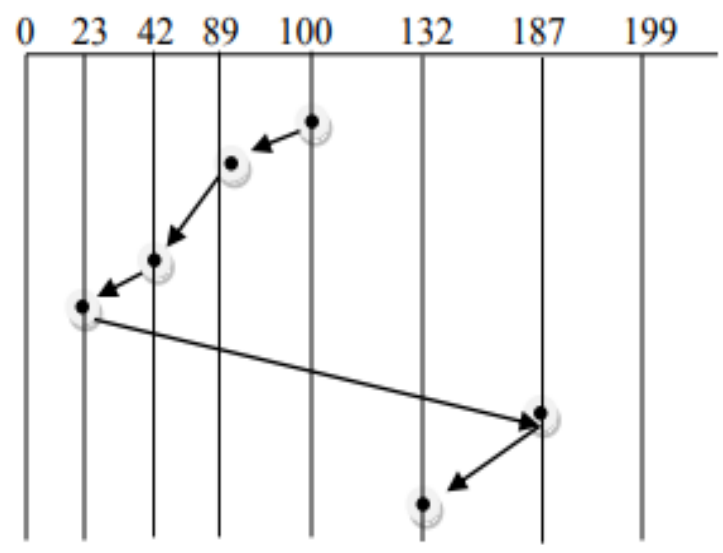

Figure.6. C-LOOKS Scheduling Principle

Overall head movements $=(100-89)+(89-42)+(42-23)+$ $(23-187)+(187-132)=296$ cylinders

Overall seek time $=296 \times 3=888 \mathrm{~ms}$

\section{E.2 Shortest-LOOKS Scheduling Principle:}

The Shortest LOOKS scheduling principle is a deviation of LOOKS scheduling principle. Essentially this principle is applied when the disk head is located in the middle of the far and end requests. This principle is intended in order to make a pronouncement that, in which bearing it should be served first. Because of the seek time is honestly depends on the seek distance, the principle should diminish the "seek distance" so that it can reduce the "seek time".

\section{RESULTS AND DISCUSSIONS}

\section{A. F-C-F-S Scheduling Principle:}

In FCFSS principle every request gets an equal chance of servicing, so no starvation will be occurred. At the other end it does not try to produce optimum seek time obviously it may not provide the best response time.

\section{B. S-S-T-F Scheduling Principle:}

SSTFS principle lessens the standard response time so that the throughput can be greater than before. At the other end SSTFS scheduling had the overhead to compute the seek time beforehand. It can cause starvation for a request if it has higher seek time than the incoming requests. This sort of approach will favor only some requests.

\section{SCANS Scheduling Principle:}

Using SCANS principle high input-output ratio and low response time can be achieved. On the other hand continuous postponement of a request for the location just visited by the disk arm which cannot be avoided.

\section{Circular-SCANS Scheduling Principle:}

Circular SCANS scheduling principle provides more unvarying response time and access time compared to SCAN scheduling principle.

\section{E. LOOKS Scheduling Principle:}

LOOKS scheduling principle provides the main benefit is that it merely performs sweeps that is large enough to service the entire requests in the request queue. With this principle the effectiveness can be improved by avoiding superfluous seek process. So that it will maximize the throughput.

\section{F. Circular-LOOKS Scheduling Principle:}

Circular LOOKS scheduling principle gives lesser response time than LOOK scheduling principle but the throughput is not pleasing.

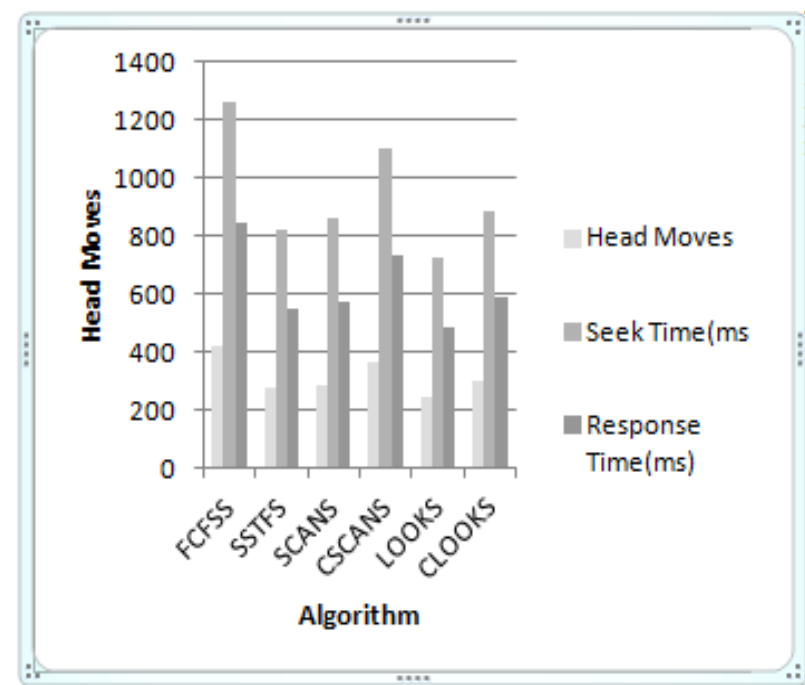

Figure.7.Seek Time and Response Time of Various Diskette Scheduling Principles

\section{CONCLUSION}

In this paper we discussed various disk scheduling principles for a selected set of $\mathrm{I} / \mathrm{O}$ request queue with a given scenario with its own merits and demerits. For the selected request queue SSTFS and LOOKS scheduling principles earns more response time when compared to the other principles. And these two principles perform more or less similar and have same number of head movements.

Here we can conclude that which principle has a reduced number of head movements and lower access time will produce higher disk response.

\section{REFERENCES}

1. Shenze Chen, John A.Stankovic "Performance Evaluation of Two New Disk Scheduling Algorithms for real-time systems", September 1991, Volume 3,Issue 3,pp 307-336[https://link.springer.com>article

2. M.R.MaheshKumar,B.RenukaRajendra," An Improved

Approach to Maximize the Performance of Disk Scheduling Algorithm by Minimizing the Head Movement and Seek Time Using Sort Mid Current Comparison (SMCC) Algorithm", Procedia Computer Science, Volume 57,2015,Page 222-231

3. Tung-Shou Chen,Wei Pang Yang,R. C. T. Lee,"Amortized analysis of some disk scheduling algorithms: SSTF, SCAN, and N-Step SCAN", BIT Numerical Mathematics,December 1992,Volume 32,Issue 4.pp 546-558

4. Andrew S Tanenbaum,"Operating Systems Design and Implementation", Sixth Edition 
5. Jogamohan Medak,Partha Pratim Gogoi,"A Comprehensive Analysis of Disk Scheduling Algorithms",International Journal Of Latest Trends in Engineering and Technology,Volume 11,Issue 1,pp 053-058

6. Abraham Sliberschatz,Peter Baer Galvin,Greg Gagne,"Operating System Concepts", Ninth Edition

7. William Stallings, "Operating Systems, Internal and Design Principal", Fifth Edition

8. Zvonco Vranesic and Safwat Zaky, "Computer Organization”, Fifth Edition

\section{AUTHOR PROFILE}

A post graduate of Anna University and Working as an Assistant Professor in the department of Computer Science and Engineering, Hindusthan College of Engg. And Tech., Coimbatore, Tamil Nadu, India. Published a paper in "Springer Link". Presented papers in various national and international conferences. 